\title{
Detailed comparison of Candida albicans and Candida glabrata biofilms under different conditions and their susceptibility to caspofungin and anidulafungin
}

\author{
Soňa Kucharíková, ${ }^{1,2,3}$ Hélène Tournu, ${ }^{1,2}$ Katrien Lagrou, ${ }^{4}$ \\ Patrick Van Dijck ${ }^{1,2}$ and Helena Bujdáková ${ }^{3}$ \\ ${ }^{1}$ Laboratory of Molecular Cell Biology, Katholieke Universiteit (KU) Leuven, Leuven, Belgium \\ ${ }^{2}$ VIB Department of Molecular Microbiology, KU Leuven, Kasteelpark Arenberg 31, Box 2438, \\ B-3001 Leuven-Heverlee, Belgium \\ ${ }^{3}$ Comenius University in Bratislava, Faculty of Natural Sciences, Department of Microbiology and \\ Virology, Mlynská dolina B-2, 84215 Bratislava, Slovak Republic \\ ${ }^{4}$ Department of Medical Diagnostic Sciences, KU Leuven, Leuven, Belgium
}

Correspondence

Helena Bujdáková

bujdakova@fns.uniba.sk

Received 7 March 2011

Accepted 6 May 2011

\section{INTRODUCTION}

Candida albicans is considered the most commonly isolated human fungal species, followed by Candida glabrata (Segireddy et al., 2011). The importance of both species has increased dramatically because of their ability to form biofilms on different types of implant (Ramage et al.,

\footnotetext{
Abbreviations: 3-D, three-dimensional; CSLM, confocal scanning laser microscopy; FM, fluorescence microscopy.

A supplementary table is available with the online version of this paper.
}

2006). In comparison with the biofilm architecture of C. albicans, which is composed of yeast cells, hyphae and pseudohyphae, C. glabrata biofilms exist as a multilayer structure of yeast cells; in both cases, the biofilms are embedded in extracellular matrix. Despite their structural diversity, biofilm formation by both Candida species is influenced by the choice of biomaterial, medium and carbohydrate source and concentration. Additionally, $\mathrm{pH}$ plays an important role, as Candida spp. may colonize different niches with respect to the environmental $\mathrm{pH}$ (Biswas \& Chaffin, 2005; Schmidt et al., 2008). To date, there is no strict recommendation for a specific medium 
suitable for in vitro biofilm experiments that would strictly resemble the composition of the human fluids surrounding the device in vivo. Recently, Uppuluri et al. (2009) characterized C. albicans biofilms in synthetic urine medium that mimics the physiological conditions during biofilm development on urinary catheters.

The development of C. albicans and C. glabrata biofilms involves the regulation of specific genes, expression of which can be affected by the environment. For instance, C. albicans adhesins Als1, Als3 and Eap1 are highly expressed in biofilms in vitro (Li et al., 2007; Nailis et al., 2010) and in vivo (Nett et al., 2009; Ričicová et al., 2010). In C. glabrata, Epa6 has been shown to be involved in mature biofilm development (Iraqui et al., 2005).

The major problem in treating biofilm-associated infections is the resistance of the fungal population to most classes of antifungals. However, caspofungin and anidulafungin were shown to have activity on mature C. albicans biofilms in vitro (Katragkou et al., 2008; Pemán et al., 2008) and in vivo (Shuford et al., 2006; Kucharíková et al., 2010a). Caspofungin was also shown to be active against mature C. glabrata biofilms formed in vitro (Choi et al., 2007).

This study provides a detailed characterization of the influence of two different culture media (RPMI 1640 and YNB) at two different $\mathrm{pHs}$ ( $\mathrm{pH} 5.6$ and 7.0) on C. albicans SC5314 and C. glabrata ATCC 2001 adhesion, biofilm development and susceptibility to echinocandins.

\section{METHODS}

Strains. For this study, C. albicans SC5314 (Gillum et al., 1984) and C. glabrata ATCC 2001 (American Type Culture Collection) were used. The strains were routinely cultivated on YPD medium (1\% yeast extract, $2 \%$ Bacto-peptone and $2 \%$ D-glucose, supplemented with $2 \%$ agar) at $37{ }^{\circ} \mathrm{C}$.

Biofilm formation. Biofilms were formed in 96-well polystyrene plates according to Ramage et al. (2001). Briefly, C. albicans and C. glabrata were incubated at $37{ }^{\circ} \mathrm{C}$ overnight on YPD plates. Cell suspensions of $1 \times 10^{6} \mathrm{cells} \mathrm{ml}^{-1}$ were prepared for both species by counting in RPMI 1640 medium (with glutamine and phenol red, without bicarbonate) buffered with MOPS (Sigma), or in YNB (Yeast Nitrogen Base with amino acids and ammonium sulfate; Difco) buffered with $0.05 \mathrm{M} \mathrm{KH}_{2} \mathrm{PO}_{4} / \mathrm{Na}_{2} \mathrm{HPO}_{4}$. Both media were supplemented with D-glucose ( $2 \%$ final). Of note, RPMI 1640 medium itself contains $0.2 \%$ D-glucose and therefore this medium was supplemented with D-glucose up to $2 \%$ as a final concentration. A supplementary table showing detailed composition of RPMI 1640 and YNB media used in this study is available in JMM Online. The $\mathrm{pH}$ was adjusted to 5.6 or 7.0. In RPMI 1640 medium, pH 7.0 was achieved with $1 \mathrm{M} \mathrm{NaOH}$, according to the NCCLS M27-A3 protocol (NCCLS, 2008). In the same medium, the $\mathrm{pH}$ was adjusted to 5.6 with a few drops of $1 \mathrm{M} \mathrm{HCl}$. Inoculum of each strain $(100 \mu \mathrm{l})$ was added to the flat-bottom 96-well polystyrene plate in quadruplicate. After a period of adhesion $(90 \mathrm{~min})$ at $37^{\circ} \mathrm{C}$, non-attached Candida cells were removed by two rounds of washing with $1 \times$ PBS. One set of plates was analysed immediately after the period of adhesion. A second set of plates was incubated at $37{ }^{\circ} \mathrm{C}$ for an additional $48 \mathrm{~h}$ in fresh medium. Mature biofilms ( $48 \mathrm{~h}$ ) were washed twice with $1 \times$ PBS. Remaining cells were evaluated for their metabolic activity by the reduction of XTT [2,3-bis(2-methoxy-4-nitro-5-sulfophenyl)- $2 \mathrm{H}$ - tetrazolium-5-carboxanilide], as described by Ramage et al. (2001). Each assay in quadruplicate was reproduced five times.

The mature $(48 \mathrm{~h})$ C. albicans and C. glabrata biofilms used for fluorescence microscopy (FM) and confocal scanning laser microscopy (CSLM) were developed on highly adhesive, round tissueculture coverslips (diameter $13 \mathrm{~mm}$; Sarstedt). One millilitre of cell suspension $\left(1 \times 10^{6}\right.$ cells $\left.\mathrm{ml}^{-1}\right)$ prepared in RPMI 1640 or YNB medium at $\mathrm{pH} 5.6$ or 7.0 was added to the wells, each containing a coverslip, and incubated at $37{ }^{\circ} \mathrm{C}$ for $90 \mathrm{~min}$. Then, the coverslips were gently washed twice with $1 \mathrm{ml} 1 \times$ PBS, placed into a clean 24well tissue-culture plate and covered with fresh medium for an additional $48 \mathrm{~h}$. The mature biofilms were further washed twice with $1 \times$ PBS before visualization.

FM. Visualization of the attached biofilms and of the morphological composition of the biofilm top layer was carried out by FM. Intact coverslips with attached biofilms were transferred to clean 24-well tissue-culture plates. Each well contained $500 \mu \mathrm{l} 1 \times$ PBS with $20 \mu \mathrm{l}$ Calcofluor White $(0.05 \%$, v/v) (Sigma). Biofilm structures were observed with a Zeiss Axioplan 2 fluorescence microscope. Images were acquired with a Zeiss Axiocam HRm camera using Axiovision 3.0 software (Carl Zeiss). FM was performed three times independently. Each time, two coverslips were used per condition.

CSLM. The three-dimensional (3-D) structure of mature C. albicans and C. glabrata biofilms grown under different conditions was determined by CSLM. The biofilms formed on coverslips were transferred gently to a clean 24-well tissue-culture plate and stained with $50 \mu \mathrm{g}$ Concanavalin A-Alexa Fluor 488 conjugate $\mathrm{ml}^{-1}$ (C-11252; Molecular Probes) in PBS. The samples were incubated for $1 \mathrm{~h}$ at $37^{\circ} \mathrm{C}$ in the dark with gentle agitation. Confocal images were acquired according to Řičicová et al. (2010). The vertical ( $x z)$ sections or side views of the 3-D reconstructed images were used to determine biofilm thickness and architecture. The thickness was estimated from the outer edges of the area where fluorescent signal gain intensity was above half of its maximum. CSLM was performed twice independently. Each time, two coverslips were used per condition.

Quantitative real-time PCR. Mature (48 h) Candida biofilms were formed in six-well tissue-culture plates to obtain the necessary amount of biofilm biomass. The biofilms were scraped off the bottom of the wells by using sterile cell scrapers and washed in DEPC-treated water. Total RNA from biofilms was extracted by using a RiboPureYeast kit (AM1926; Ambion). Then, $1 \mu \mathrm{g}$ RNA was treated with amplification-grade DNase I and used for cDNA synthesis using a Reverse Transcription kit (A3500; Promega). Quantitative PCR was performed on a StepOnePlus real-time PCR system (Applied Biosystems) using a Kapa SYBR Fast kit (Kapabiosystems) according to the manufacturer's instructions. Data for each target gene were calculated and expressed as fold regulation in comparison to the reference gene ACT1 for each condition tested, using the standard curve quantification method (Livak \& Schmittgen, 2001). The following primers were used for $C$. albicans analyses: those for ACT1 and EAP1 were described previously (Řičicová et al., 2010). $A L S 1$ and $A L S 3$ primers were designed as described by Green et al. (2005). The sequence of the C. glabrata ACT1 forward primer was 5' CGCTTTGGACTTCGAACAAGAA-3' and that of the reverse primer was 5'-GTTACCGATAGTGATGACTTGAC-3'. For C. glabrata EPA6, the sequence of the forward primer was $5^{\prime}$-CAAAATCTAAACTGGCAGACAATAATG-3' and that of the reverse primer was $5^{\prime}$-AGATCAGAACCAGAACCTTGgATAATA-3' (K. De Brucker, J. Serneels, E. Boons, T. Schwarzmüller, J. Quintin, M. Henrigues, K. Kuchler, P. Van Dijck \& H. Tournu, unpublished data).

The quantitative RT-PCR assay was performed twice independently. RNA isolation and cDNA synthesis were done twice from different biofilms. 
Biofilm antifungal-susceptibility testing. Caspofungin (Merck) and anidulafungin (Pfizer) were used in this study. The stock solution of caspofungin was prepared in sterile water. Anidulafungin was prepared in $100 \%$ DMSO and kept at $-20{ }^{\circ} \mathrm{C}$ before use. Prior to the experiment, both antifungals were dissolved in corresponding sterile medium according to the NCCLS M27-A3 protocol (NCCLS, 2008).

Susceptibility testing of planktonic cells to caspofungin and anidulafungin was performed according to the NCCLS M27-A3 protocol (NCCLS, 2008). Data were determined as minimal inhibitory concentrations of the drug that inhibit fungal growth by 50 or $90 \%$ $\left(\mathrm{MIC}_{50}\right.$ or $\mathrm{MIC}_{90}$, respectively).

Mature Candida biofilms (24 h) were formed in 96-well polystyrene plates in RPMI 1640 medium, $\mathrm{pH} 5.6$ or 7.0 , or YNB medium, pH 5.6 or 7.0, as described above. Solutions of caspofungin $\left(8-0.01562 \mu \mathrm{g} \mathrm{ml}^{-1}\right)$ and anidulafungin $\left(16-0.03125 \mu \mathrm{g} \mathrm{ml}^{-1}\right)$ were added to preformed mature biofilms and incubated for an additional $24 \mathrm{~h}$. Biofilms treated only with fresh medium served as untreated controls. Measurement of biofilm cell metabolic activity using the XTT-reduction assay was performed as described above. Antifungal effect was determined by comparing the reduction in the mean $A_{490}$ of the antifungal-challenged biofilm condition to the unchallenged controls. The results are shown as sessile $\mathrm{MIC}_{50}$ and $\mathrm{MIC}_{90}$ values $\left(\mathrm{SMIC}_{50}\right.$ and $\mathrm{SMIC}_{90}$, respectively). These values determine the minimal inhibitory concentration of the drug that inhibits the growth of sessile cells by 50 and $90 \%$, respectively. Each assay was performed in quadruplicate each time and repeated three times.

Statistical analyses. For the statistical analyses, Student's $t$-test was used. Results were considered to be statistically significant when $P<0.05$.

\section{RESULTS}

\section{Medium composition modulates adherence and biofilm formation more than $\mathrm{pH}$ in C. glabrata and C. albicans}

Candida biofilm development in the host is affected by variable and sometimes changing environmental conditions, depending on the infection site. Many studies in vitro have reported the use of a multitude of media, substrates and techniques to study biofilms (Biswas \& Chaffin, 2005; Schmidt et al., 2008; Uppuluri et al., 2009). Here, we compared the effect of two commonly used media, namely RPMI 1640 and $\mathrm{YNB}$, at two different $\mathrm{pH}$ values ( $\mathrm{pH} 5.6$ and 7.0) on C. glabrata and C. albicans adhesion and mature biofilm development. Biofilms were formed on polystyrene and assessed by the XTT-reduction assay measured at $490 \mathrm{~nm}$. The results are shown in Fig. 1 and are determined as the mean \pm SD of the metabolic activity of adhering or biofilm-forming cells. C. glabrata adhesion properties were independent of the choice of medium or $\mathrm{pH} \quad(P>0.05) \quad$ (Fig. 1a), whereas the effect of growth conditions on the ability of $C$. albicans to adhere was more pronounced (Fig. 1c). C. albicans manifested significantly better adhesion properties when grown in RPMI 1640 medium, compared with its adhesion in YNB medium $(P<0.05)$. Moreover, in RPMI 1640 medium at $\mathrm{pH} 7.0$, C. albicans had significantly enhanced adhesion capabilities compared with those at pH $5.6\left(A_{490}=0.566 \pm 0.108\right.$ and
$0.924 \pm 0.209$, respectively) $(P<0.05)$. However, the $\mathrm{pH}$ did not have a significant effect on $C$. albicans adhesion in YNB $(P>0.05)$. The metabolic activity of $C$. albicans mature biofilm-forming cells was significantly reduced in YNB medium $\left(A_{490}=0.530 \pm 0.072\right.$ and $0.660 \pm 0.031$, respectively) compared with RPMI 1640 medium $\left(A_{490}=1.240\right.$ \pm 0.188 and $1.410 \pm 0.086$, respectively) $(P<0.05)$ (Fig. $1 \mathrm{~d}$ ). Despite the fact that $\mathrm{pH} 7.0$ enhanced the yeast-to-hyphae transition, it was not shown to have a significant effect on a mature $C$. albicans biofilm $(P>0.05)$. Considering C. glabrata biofilm formation (Fig. 1b), the medium is a crucial factor during its maturation. C. glabrata biofilmforming cells demonstrated significantly less metabolic activity when grown in YNB medium $\left(A_{490}=0.360 \pm 0.075\right.$ and $0.453 \pm 0.044$, respectively) compared with the results obtained after growth in RPMI 1640 medium $\left(A_{490}=1.310\right.$ \pm 0.272 and $1.483 \pm 0.274$, respectively) $(P<0.05)$. Similar to adhesion, C. glabrata biofilm development was not influenced by the $\mathrm{pH}$ tested $(P>0.05)$.

\section{RPMI 1640 medium promotes the development of mature biofilms and induces the yeast-to-hyphae transition in C. albicans}

C. albicans biofilms are known to be composed of yeast and hyphal cells, building a layered, attached structure (Ramage et al., 2006). Therefore, we examined the morphology of the cells within a mature biofilm ( $48 \mathrm{~h}$ ) by FM and the biofilm thickness with CSLM for the four conditions tested. The biofilm architecture of $C$. albicans and C. glabrata is illustrated in Fig. 2. The measurements of biofilm thickness are displayed in Table 1. The choice of the different media influenced the biofilm thickness and, additionally, the $\mathrm{pH}$ contributed to the yeast-to-hyphae transition in C. albicans.

In comparison with the biofilms formed in RPMI 1640 medium (Fig. 2c, d), C. glabrata cells manifested adhesion rather than multilayer biofilm architecture in YNB medium, regardless of the $\mathrm{pH}$ (Fig. 2a, b). Therefore, in this particular case, the thickness could not be estimated reliably. On the other hand, in RPMI 1640 medium at pH 5.6, C. glabrata cells formed scattered biofilm layers, with some areas of the substrate remaining uncovered (Fig. 2c). The thickness of the mature biofilms on covered places was approximately $20 \mu \mathrm{m}$. Despite this, in the same medium, but at $\mathrm{pH} 7.0$, the polystyrene surface was fully covered with yeast cells and the thickness of the mature biofilm increased to approximately $25 \mu \mathrm{m}$ (Fig. 2d). Similarly to C. glabrata, C. albicans manifested adhesion rather than biofilm architecture when grown in YNB medium at both pHs (Fig. 2e). Although $\mathrm{pH} 7.0$ enhanced the yeast-to-hyphae transition, which is an important factor for C. albicans multilayer biofilm development, the polystyrene remained unevenly covered (Fig. 2f). These patches of Candida cells were measured and their thickness was revealed to be approximately $50 \mu \mathrm{m}$. On the other hand, C. albicans biofilms formed in RPMI 1640 medium at pH 5.6 or 7.0 were composed mainly of hyphae (Fig. 2g, h). During biofilm development in RPMI 1640 

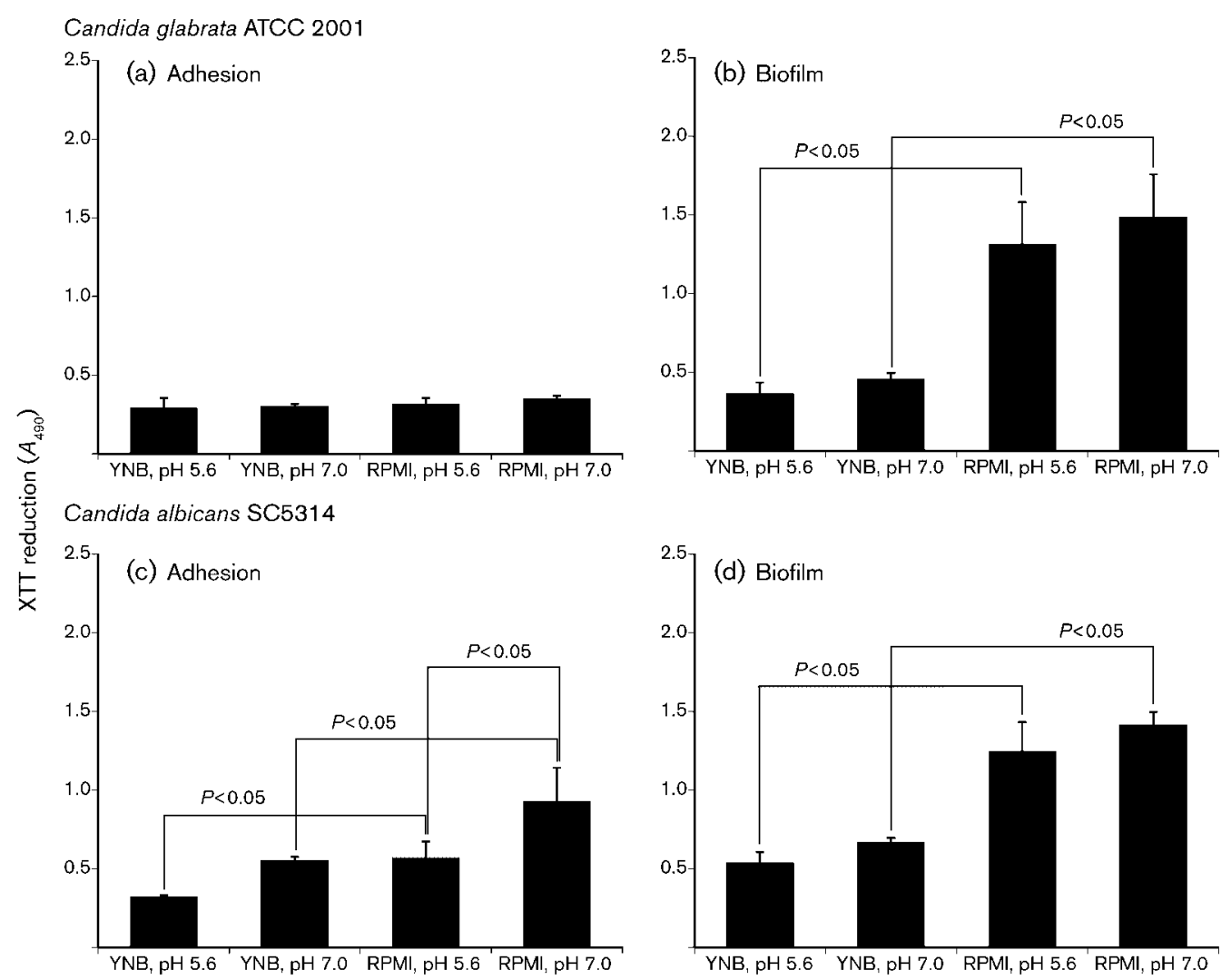

Fig. 1. RPMI 1640 medium significantly promotes biofilm formation in C. glabrata ATCC 2001 and C. albicans SC5314. An XTT-reduction assay, determined at $A_{490}$, was used as a measure of metabolic activity of C. glabrata ATCC 2001 (a, b) and C. albicans SC5314 (c, d) cells during adhesion and biofilm formation on 96-well polystyrene plates in RPMI 1640-MOPS and YNB media, at $\mathrm{pH} 5.6$ or 7.0. The XTT measurements were performed after a period of adhesion $\left(90 \mathrm{~min}\right.$ at $\left.37^{\circ} \mathrm{C}\right)$, whilst biofilms were quantified after $48 \mathrm{~h}$ at $37{ }^{\circ} \mathrm{C}$. The results were considered as statistically significant when $P<0.05$. SD was calculated from five independent experiments. Each condition was tested in quadruplicate.

Candida glabrata ATCC 2001
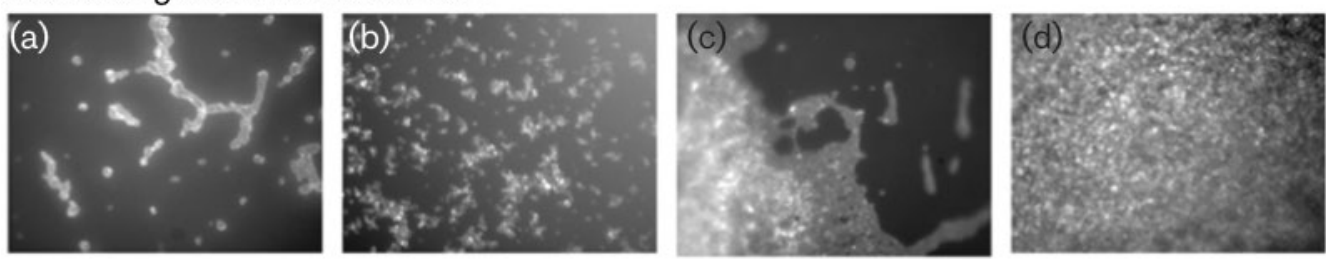

\section{Candida albicans SC5314}

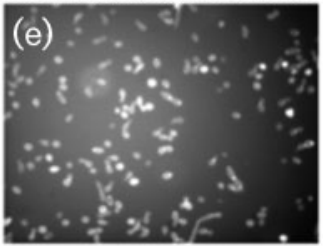

YNB, pH 5.6

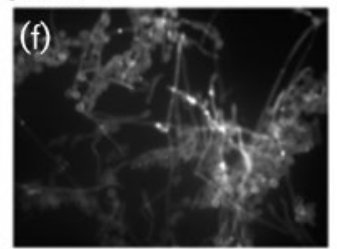

YNB, pH 7.0

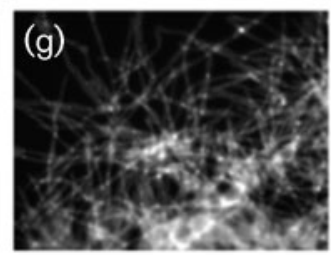

RPMl 1640, pH 5.6

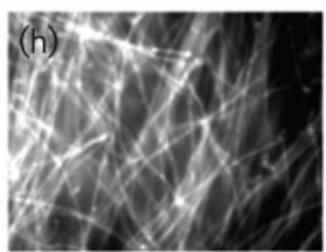

RPMl 1640, pH 7.0

Fig. 2. Growth medium and $\mathrm{pH}$ influence cell morphology and biofilm thickness. FM images display the top layers of $48 \mathrm{~h}$-old biofilms developed on polystyrene coverslips. Mature C. glabrata ATCC 2001 (a-d) and mature C. albicans SC5314 (e-h) biofilms were stained with Calcofluor White prior to visualization. Magnification, $\times 40$. FM was performed three times on two coverslips used per condition. 
Table 1. Thickness of mature C. glabrata ATCC 2001 and C. albicans SC5314 biofilms as determined by CSLM

Approximate values of biofilm thickness, represented in $\mu \mathrm{m}$, were obtained from vertical sections through 3-D confocal images.

\begin{tabular}{|lcc|}
\hline Growth conditions & C. glabrata ATCC 2001 & C. albicans SC5314 \\
\hline YNB, pH 5.6 & $10-15$ & $29-34$ \\
YNB, pH 7.0 & $10-15$ & 51 \\
RPMI 1640, pH 5.6 & 20 & 60 \\
RPMI 1640, pH 7.0 & 25 & 100 \\
\hline
\end{tabular}

medium at $\mathrm{pH} 5.6$, the biofilm thickness was approximately $60 \mu \mathrm{m}$ (Fig. 2g), whereas in the same medium, but at $\mathrm{pH}$ 7.0, the thickness reached approximately $100 \mu \mathrm{m}$ (Fig. $2 \mathrm{~h})$. It is noteworthy to mention that the observations of hazy material covering C. albicans or C. glabrata biofilms formed in RPMI 1640-MOPS medium (Fig. 2c, d, g, f) suggest the presence of extracellular matrix.

These results demonstrate that RPMI 1640 medium induces the yeast-to-hyphae transition in C. albicans, resulting in thicker biofilms compared with growth in YNB medium. In addition, we showed that C. glabrata forms multilayer biofilms on polystyrene only in the presence of RPMI 1640 medium at $\mathrm{pH}$ 7.0.

\section{Transcriptional expression of adhesin genes correlates positively with biofilm formation}

We anticipated that the expression profile of Candida biofilm-associated genes may change due to exposure to different environmental conditions. We therefore analysed the changes in expression of genes participating significantly in adhesion and biofilm formation, such as C. glabrata EPA6 (Iraqui et al., 2005) and C. albicans ALS1, ALS3 and EAP1 (Nailis et al., 2010; Nobile et al., 2006). The results are displayed in Figs 3 and 4(a, b, c), respectively. The transcript profile of EPA6 was induced significantly in cells growing in RPMI 1640-MOPS medium compared with YNB medium, regardless of the $\mathrm{pH}$ used $(P<0.05)$ (Fig. 3). Additionally, significant upregulation was observed in the same medium at pH $7.0(P<0.05)$ (Fig. 3). For comparison, we examined the EPA6 expression profile from $48 \mathrm{~h}$-old planktonic cultures (stationary phase) in RPMI 1640 or YNB medium at $\mathrm{pH} 5.6$ or 7.0. Neither RPMI 1640 medium nor the $\mathrm{pH}$ promoted EPA6 expression, compared with YNB medium when cells were grown in liquid cultures (data not shown), indicating that the results obtained from sessile populations are biofilm-specific.

Further, we examined the changes in the expression profile of C. albicans adhesion-associated genes such as ALS1, ALS3 and EAP1. The results are displayed in Fig. 4(a, b, c), respectively. None of the three genes analysed was regulated in YNB-grown biofilms, regardless of the $\mathrm{pH}$. On the contrary, RPMI 1640 medium influenced the expression of these adhesins in a pH-dependent manner. The ALS3 gene

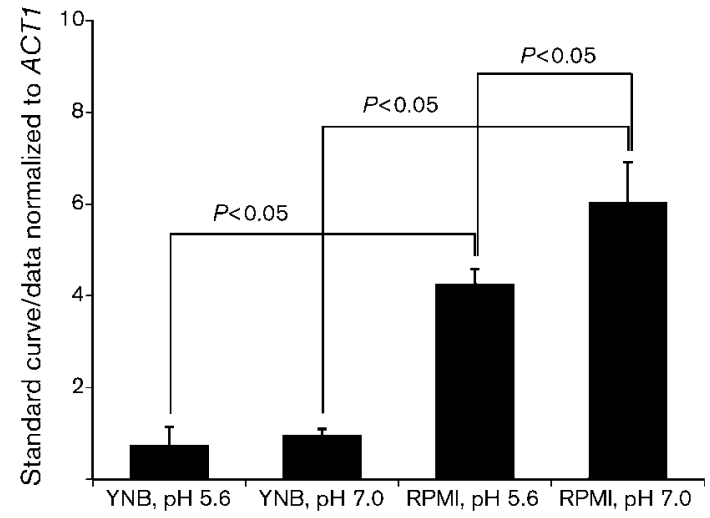

Fig. 3. EPA6 in C. glabrata ATCC 2001 is upregulated significantly in RPMI 1640 medium. Expression analyses of the adhesion-encoding gene in mature $(48 \mathrm{~h})$ C. glabrata biofilms developed in RMPI 1640 or YNB medium at $\mathrm{pH} 5.6$ or 7.0 are shown. The data were normalized to ACT1 using the standard curve quantification method. The increased expression levels of EPA6 were considered as statistically significant when $P<0.05$. SD was calculated from two independent experiments on different occasions.

was upregulated significantly at $\mathrm{pH} 7.0$ compared with pH 5.6 and in YNB-grown biofilms $(P<0.05)$ (Fig. 4b). A similar pattern of expression was observed for the EAP1 gene $(P<0.05)$ (Fig. 4c). In contrast, ALS1 expression was not promoted by the conditions tested (Fig. 4a). It is noteworthy to mention that the data demonstrated above were normalized to ACT1; it is known that the expression of this gene may change during morphogenesis (Delbrück \& Ernst, 1993; Michán \& Pueyo, 2009), which is characteristic for C. albicans during biofilm development. Because of this, in addition, the data were also normalized to TEF1. Regardless of the housekeeping gene used, the expression levels of ALS1, $A L S 3$ and EAP1 were identical to those observed above (data not shown). This observation excludes the potential influence of housekeeping-gene expression on the evaluation of the expression of tested genes.

\section{RPMI 1640-grown biofilms are less susceptible to echinocandins than YNB-grown biofilms in C. albicans, but not in C. glabrata}

The results mentioned above led us to the assumption that the increased biofilm thickness observed for C. albicans and C. glabrata grown in RPMI 1640 medium at pH 5.6 and 7.0 might have an influence on the efficiency of antifungal drugs against biofilms. Hence, we tested the effect of two echinocandins, caspofungin and anidulafungin, against biofilms formed under the different environmental conditions. C. albicans and C. glabrata planktonic cell cultures were susceptible to both echinocandins (Table 2). Following antifungal treatment, biofilms were assessed by XTT measurements, and the data are displayed in Table 3. Both caspofungin and anidulafungin were shown to be very 

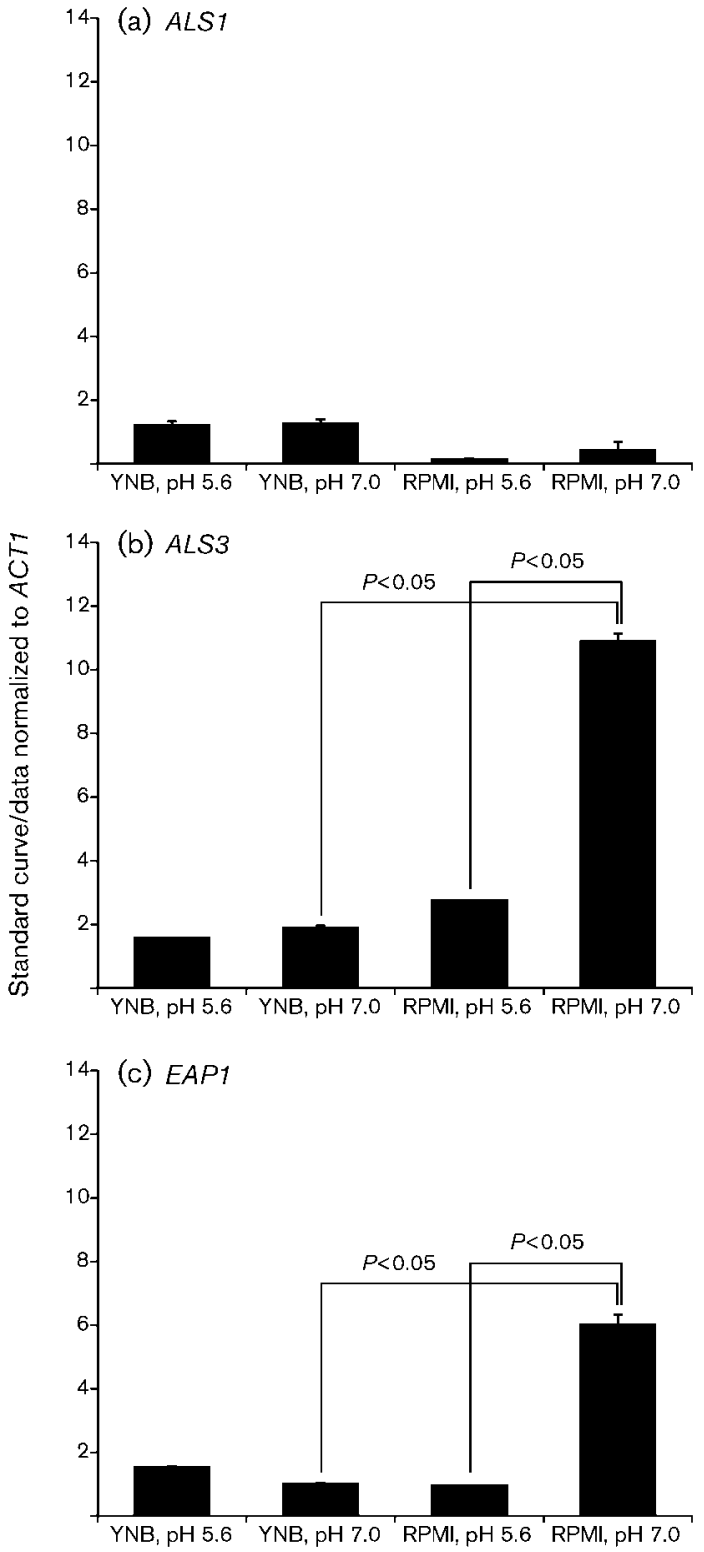

Fig. 4. $A L S 3$ and EAP1 in C. albicans SC5314 are highly expressed under the most biofilm-favourable growth conditions. The expression level of adhesion-encoding genes ALS1 (a), ALS3 (b) and EAP1 (c) in mature (48 h) C. glabrata and C. albicans biofilms developed in RMPI 1640 or YNB medium at $\mathrm{pH} 5.6$ or 7.0 is shown. All expression levels are shown as relative fold expression compared with the ACT1 level using the standard curve quantification method. SD was calculated from two independent experiments on different occasions.

potent against C. albicans mature biofilms under all conditions tested, as indicated by the low $\mathrm{SMIC}_{50}$ and SMIC $_{90}$ values, whilst C. glabrata biofilms were slightly less susceptible. For both species, the highest $\mathrm{SMIC}_{90}$ values were always observed in the condition promoting the thickest biofilms - RPMI 1640 medium at $\mathrm{pH}$ 7.0. This reduced susceptibility to the antifungal treatment was also observed on biofilms grown in RPMI 1640 medium at $\mathrm{pH} 5.6$, but to a lower extent. In conclusion, YNB-grown biofilms seem more susceptible to echinocandins than RPMI 1640-grown biofilms.

\section{DISCUSSION}

This study gives a detailed comparison between the biofilms formed by C. albicans SC5314 and C. glabrata ATCC 2001 grown in RPMI 1640 and YNB media. We also elucidated the effect of $\mathrm{pH} 5.6$ and 7.0 during biofilm development, because adaptation to changes in $\mathrm{pH}$ during biofilm formation is crucial not only for its development, but also for survival of microbial communities at colonized host sites (Schmidt et al., 2008) as well as on medical devices (Ramage et al., 2006). The two media tested, YNB and RPMI 1640, have remained favourite choices for in vitro biofilm formation (Biswas \& Chaffin, 2005; Cateau et al., 2008; Chandra et al., 2001). YNB medium is less nutrient-rich for growth of planktonic cells than RPMI 1640 medium, which mimics the composition of human fluids (Chandra et al., 2005). This medium was used previously for $C$. albicans attachment on polyurethane prior to subcutaneous catheter implantation in vivo (Řičicová et al., 2010). Moreover, RPMI 1640 is the standard medium for in vitro susceptibility testing of planktonic cells to antifungals according to the NCCLS M27-A3 protocol (NCCLS, 2008). However, there are no studies that have focused strictly on the testing of RPMI 1640 and YNB media on the different steps of biofilm development.

From our study, it is clear that the medium and $\mathrm{pH}$ tested did not have any effect on the C. glabrata adhesion process, whereas the ability of $C$. albicans to adhere was significantly higher in RPMI 1640 than in YNB medium. This observation could be explained by the induced $C$. albicans yeastto-hyphae transition process, particularly in RPMI 1640 medium. The yeast-to-hyphae transition has been considered one of the crucial factors involved in C. albicans attachment to a surface (Blankenship \& Mitchell, 2006). Neutral pH promotes the yeast-to-hyphae transformation and contributes to better $C$. albicans adhesion in our assay in RPMI 1640 medium. In contrast, C. glabrata is not able to switch from yeast to hyphae, but it was able to form a multilayer, highly potent biofilm, as described previously (Kucharíková et al., 2010b; Nikawa et al., 1997). In addition to cell morphology, the thickness of the biofilms also showed great variation. Both C. glabrata and C. albicans formed significantly thicker biofilms in RPMI 1640 medium, whereas in YNB medium, cells proliferated in an adherent manner rather than forming a multilayer biofilm. Strikingly, RPMI 1640 medium seems to be superior for biofilms formed by both species. In addition, $\mathrm{pH} 7.0$ helped to promote a dense biofilm structure composed of a basal layer of yeast cells followed by hyphae in C. albicans, or a multilayer structure of the yeast cells in C. glabrata.

Because of the striking effect of the different media on biofilm formation and architecture in both Candida 
Table 2. Susceptibility testing of C. glabrata ATCC 2001 and C. albicans SC5314 planktonic cells to caspofungin and anidulafungin

$\mathrm{MIC}_{50}$ and $\mathrm{MIC}_{90}$ values are shown as $\mu \mathrm{g} \mathrm{ml}^{-1}$.

\begin{tabular}{|lcclll|}
\hline \multirow{2}{*}{$\begin{array}{l}\text { Antifungal } \\
\text { agent }\end{array}$} & \multicolumn{2}{c}{ C. glabrata } & ATCC 2001 & & \multicolumn{2}{c|}{ C. albicans } & SC5314 \\
\cline { 2 - 3 } \cline { 6 - 6 } & MIC $_{\mathbf{5 0}}$ & MIC $_{\mathbf{9 0}}$ & & MIC $_{\mathbf{5 0}}$ & MIC $_{\mathbf{9 0}}$ \\
\hline Caspofungin & 0.25 & 0.5 & & 0.125 & 0.125 \\
Anidulafungin & 0.5 & 0.5 & & 0.0625 & 0.0625 \\
\hline
\end{tabular}

species, we investigated the expression profile of the C. glabrata EPA6 gene and the C. albicans ALS1, ALS3 and EAP1 genes, all involved directly or indirectly in biofilm formation. It was demonstrated previously that C. glabrata EPA6 is transcribed at the highest level during the stationary-growth phase, in contrast to other EPA genes, and that cells in the stationary phase adhered better than metabolically active cells (Iraqui et al., 2005). In all of our assays, C. glabrata cultures were grown up to the stationary phase to ensure that the medium contained totally adherent cell populations. We observed an upregulation of C. glabrata EPA6 during biofilm formation in RPMI 1640 medium at both pHs. This upregulation of EPA6 correlates well with the increased thickness and viability of the biofilms developed in the same medium.

During C. albicans biofilm development, ALS1, ALS3 and EAP1 play roles in different stages of biofilm formation. Yeater et al. (2007) demonstrated, by microarray analyses of biofilms, that the expression of ALS1 increases during the early stages of biofilm development (6-12 h) and declines at the later stages. This documented profile of expression could explain why we did not observe any regulation of $A L S 1$ under any of the conditions tested, as biofilms were grown for $48 \mathrm{~h}$ before analyses. However, the data are controversial for that gene, as it is expressed differentially depending on growth in vitro (García-Sánchez et al., 2004; Nailis et al.,
2009) or in vivo (Nett et al., 2009; Ričicová et al., 2010) and also depending on the growth conditions. In our assays, the C. albicans ALS3 and EAP1 genes were upregulated in an ascending manner from $\mathrm{pH} 5.6$ to 7.0 in RPMI 1640 medium.

Both caspofungin and anidulafungin were effective against C. albicans mature biofilms, regardless of the environmental conditions used. Similarly to our study, the data of Jacobson et al. (2008) showed the $\mathrm{SMIC}_{50}$ for anidulafungin to be $\leqslant 0.03125 \mu \mathrm{g} \mathrm{ml}^{-1}$ in 28 of 30 C. albicans clinical isolates tested when the biofilm was formed in RPMI 1640 medium. In contrast to the work of Katragkou et al. (2008), we did not observe any indication of paradoxical growth during caspofungin activity against a mature $C$. albicans biofilm, even when high concentrations $\left(4-8 \mu \mathrm{g} \mathrm{ml}^{-1}\right)$ of the drug were administered to biofilms (data not shown). C. glabrata cells forming mature biofilm were less susceptible than the diploid species to caspofungin and anidulafungin in both media tested, albeit with up to half the thickness of C. albicans biofilms. In a previous study, C. glabrata bloodstream clinical isolates displayed a median caspofungin $\mathrm{SMIC}_{90}$ of $1 \mu \mathrm{g} \mathrm{ml}^{-1}$ (Choi et al., 2007), similar to data that we obtained. Both C. albicans and C. glabrata showed reduced susceptibility to echinocandins when biofilms were formed in RPMI 1640 medium compared with YNB medium, which corresponded with denser biofilm structures. The $\mathrm{pH}$ was not shown to have a significant effect on antifungal susceptibility of the cells within the biofilm.

In conclusion, C. albicans and C. glabrata biofilms displayed heterogeneous architecture, expression profiles and susceptibility to caspofungin and anidulafungin when formed under diverse environmental conditions. The medium and its $\mathrm{pH}$ influenced adhesion and biofilm development in different manners. Therefore, it is important to clearly define growth conditions prior to and during the biofilm experimental set-up. It is also essential to mimic physiological conditions when performing biofilm formation in vitro. In particular, RPMI 1640 medium contributed to the development of thick biofilm structures in both C. albicans

Table 3. Antifungal-susceptibility testing of C. glabrata ATCC 2001 and C. albicans SC5314 biofilms to caspofungin and anidulafungin

$\mathrm{SMIC}_{50}$ and $\mathrm{SMIC}_{90}$ values are shown as $\mu \mathrm{g} \mathrm{ml}^{-1}$.

\begin{tabular}{|c|c|c|c|c|c|}
\hline \multirow[t]{2}{*}{ Antifungal agent } & \multirow[t]{2}{*}{ Growth conditions } & \multicolumn{2}{|c|}{ C. glabrata ATCC 2001} & \multicolumn{2}{|c|}{ C. albicans SC5314 } \\
\hline & & $\mathrm{SMIC}_{50}$ & $\mathrm{SMIC}_{90}$ & $\mathrm{SMIC}_{50}$ & $\mathrm{SMIC}_{90}$ \\
\hline \multirow{3}{*}{ Caspofungin } & YNB, pH 7.0 & 1 & 1 & 0.25 & 0.25 \\
\hline & RPMI 1640, pH 5.6 & 2 & 2 & 0.25 & 0.5 \\
\hline & RPMI 1640, pH 7.0 & 1 & 2 & 0.5 & 1 \\
\hline \multirow{2}{*}{ Anidulafungin } & RPMI 1640, pH 5.6 & 2 & 2 & 0.0625 & 0.5 \\
\hline & RPMI 1640, pH 7.0 & 1 & 2 & 0.0625 & 0.5 \\
\hline
\end{tabular}


and C. glabrata. Moreover, during C. albicans biofilm development, the choice of a $\mathrm{pH}$ of 7.0 contributed to a faster yeast-to-hyphae transition, which resulted in denser biofilm structures compared with those formed in YNB medium. It is important to take into consideration that different environmental conditions will affect not only biofilm architecture, but also its susceptibility to antifungal agents, which underscores the need for standardized methods.

\section{ACKNOWLEDGEMENTS}

Authors' contributions: S. K. and H. T. participated in the design of the study. S.K. performed all experimental procedures and carried out the data analyses, and drafted the manuscript. H.T. helped to correct the manuscript. K. L. provided pure substances of anidulafungin and corrected the manuscript. P.V.D. helped in the design of the study and corrected the manuscript. H. B. participated in the coordination of the study and helped to correct the drafted manuscript. All authors read and approved the manuscript. This work was supported by the Slovak Ministry of Education (grant VEGA 1/0396/10), the Fund for Scientific Research Flanders (WO.004.06N) and Pfizer (grant 8960). We thank Dr D. Chorvát, $\mathrm{Jr}$, for assistance during CSLM and N. Van Vangoethem for assistance with the figures and tables.

\section{REFERENCES}

Biswas, S. K. \& Chaffin, W. L. (2005). Anaerobic growth of Candida albicans does not support biofilm formation under similar conditions used for aerobic biofilm. Curr Microbiol 51, 100-104.

Blankenship, J. R. \& Mitchell, A. P. (2006). How to build a biofilm: a fungal perspective. Curr Opin Microbiol 9, 588-594.

Cateau, E., Rodier, M. H. \& Imbert, C. (2008). In vitro efficacies of caspofungin or micafungin catheter lock solutions on Candida albicans biofilm growth. J Antimicrob Chemother 62, 153-155.

Chandra, J., Kuhn, D. M., Mukherjee, P. K., Hoyer, L. L., McCormick, T. \& Ghannoum, M. A. (2001). Biofilm formation by the fungal pathogen Candida albicans: development, architecture, and drug resistance. J Bacteriol 183, 5385-5394.

Chandra, J., Patel, J. D., Li, J., Zhou, G., Mukherjee, P. K., McCormick, T. S., Anderson, J. M. \& Ghannoum, M. A. (2005). Modification of surface properties of biomaterials influences the ability of Candida albicans to form biofilms. Appl Environ Microbiol 71, 8795-8801.

Choi, H. W., Shin, J. H., Jung, S. I., Park, K. H., Cho, D., Kee, S. J., Shin, M. G., Suh, S. P. \& Ryang, D. W. (2007). Species-specific differences in the susceptibilities of biofilms formed by Candida bloodstream isolates to echinocandin antifungals. Antimicrob Agents Chemother 51, 1520-1523.

Delbrück, S. \& Ernst, J. F. (1993). Morphogenesis-independent regulation of actin transcript levels in the pathogenic yeast Candida albicans. Mol Microbiol 10, 859-866.

García-Sánchez, S., Aubert, S., Iraqui, I., Janbon, G., Ghigo, J. M. \& d'Enfert, C. (2004). Candida albicans biofilms: a developmental state associated with specific and stable gene expression patterns. Eukaryot Cell 3, 536-545.

Gillum, A. M., Tsay, E. Y. \& Kirsch, D. R. (1984). Isolation of the Candida albicans gene for orotidine- 5 '-phosphate decarboxylase by complementation of S. cerevisiae ura3 and E. coli pyrF mutations. Mol Gen Genet 198, 179-182.
Green, C. B., Zhao, X., Yeater, K. M. \& Hoyer, L. L. (2005). Construction and real-time RT-PCR validation of Candida albicans PALS-GFP reporter strains and their use in flow cytometry analysis of ALS gene expression in budding and filamenting cells. Microbiology 151, 1051-1060.

Iraqui, I., Garcia-Sanchez, S., Aubert, S., Dromer, F., Ghigo, J. M., d'Enfert, C. \& Janbon, G. (2005). The Yak1p kinase controls expression of adhesins and biofilm formation in Candida glabrata in a Sir4p-dependent pathway. Mol Microbiol 55, 1259-1271.

Jacobson, M. J., Piper, K. E., Nguyen, G., Steckelberg, J. M. \& Patel, R. (2008). In vitro activity of anidulafungin against Candida albicans biofilms. Antimicrob Agents Chemother 52, 2242-2243.

Katragkou, A., Chatzimoschou, A., Simitsopoulou, M., Dalakiouridou, M., Diza-Mataftsi, E., Tsantali, C. \& Roilides, E. (2008). Differential activities of newer antifungal agents against Candida albicans and Candida parapsilosis biofilms. Antimicrob Agents Chemother 52, 357-360.

Kucharíková, S., Tournu, H., Holtappels, M., Van Dijck, P. \& Lagrou, K. (2010a). In vivo efficacy of anidulafungin against mature Candida albicans biofilms in a novel rat model of catheter-associated candidiasis. Antimicrob Agents Chemother 54, 4474-4475.

Kucharíková, S., Van Dijck, P., Lisalová, M. \& Bujdáková, H. (2010b). Effect of antifungals on itraconazole resistant Candida glabrata. Cent Eur J Biol 5, 318-323.

Li, F., Svarovsky, M. J., Karlsson, A. J., Wagner, J. P., Marchillo, K., Oshel, P., Andes, D. \& Palecek, S. P. (2007). Eaplp, an adhesin that mediates Candida albicans biofilm formation in vitro and in vivo. Eukaryot Cell 6, 931-939.

Livak, J. K. \& Schmittgen, T. D. (2001). Analysis of relative gene expression data using real-time quantitative PCR and the $2^{-\Delta \Delta C_{t}}$ method. Methods 25, 402-408.

Michán, C. \& Pueyo, C. (2009). Growth phase-dependent variations in transcript profiles for thiredoxin- and glutathione-dependent redox systems followed by budding and hyphal Candida albicans cultures. FEMS Yeast Res 9, 1078-1090.

Nailis, H., Vandenbroucke, R., Tilleman, K., Deforce, D., Nelis, H. \& Coenye, T. (2009). Monitoring ALS1 and ALS3 gene expression during in vitro Candida albicans biofilm formation under continuous flow conditions. Mycopathologia 167, 9-17.

Nailis, H., Kucharíková, S., Ricicová, M., Van Dijck, P., Deforce, D., Nelis, H. \& Coenye, T. (2010). Real-time PCR expression profiling of genes encoding potential virulence factors in Candida albicans biofilms: identification of model-dependent and -independent gene expression. BMC Microbiol 10, 114.

NCCLS (2008). Reference method for broth dilution antifungal susceptibility testing of yeast, approved standard M27-A3. Wayne, PA: National Committee for Clinical Laboratory Standards. http:// www.clsi.org/source/orders/free/M27-A3.pdf

Nett, J. E., Lepak, A. J., Marchillo, K. \& Andes, D. R. (2009). Time course global gene expression analysis of an in vivo Candida biofilm. J Infect Dis 200, 307-313.

Nikawa, H., Nishimura, H., Hamada, T., Kumagai, H. \& Samaranayake, L. P. (1997). Effects of dietary sugars and saliva and serum on Candida bioflim formation on acrylic surfaces. Mycopathologia 139, 87-91.

Nobile, C. J., Andes, D. R., Nett, J. E., Smith, F. J., Yue, F., Phan, Q. T., Edwards, J. E., Filler, S. G. \& Mitchell, A. P. (2006). Critical role of Bcr1-dependent adhesins in C. albicans biofilm formation in vitro and in vivo. PLoS Pathog 2, e63.

Pemán, J., Cantón, E. \& Valentín, A. (2008). Activity of anidulafungin against Candida biofilms. Rev Iberoam Micol 25, 124-128 (in Spanish). 
Ramage, G., Vandewalle, K., Wickes, B. L. \& López-Ribot, J. L. (2001). Characteristics of biofilm formation by Candida albicans. Rev Iberoam Micol 18, 163-170.

Ramage, G., Martínez, J. P. \& López-Ribot, J. L. (2006). Candida biofilms on implanted biomaterials: a clinically significant problem. FEMS Yeast Res 6, 979-986.

Řičicová, M., Kucharíková, S., Tournu, H., Hendrix, J., Bujdáková, H., Van Eldere, J., Lagrou, K. \& Van Dijck, P. (2010). Candida albicans biofilm formation in a new in vivo rat model. Microbiology 156, 909919.

Schmidt, P., Walker, J., Selway, L., Stead, D., Yin, Z., Enjalbert, B., Weig, M. \& Brown, A. J. (2008). Proteomic analysis of the $\mathrm{pH}$ response in the fungal pathogen Candida glabrata. Proteomics 8, 534-544.
Segireddy, M., Johnson, L. B., Szpunar, S. M. \& Khatib, R. (2011). Differences in patient risk factors and source of candidaemia caused by Candida albicans and Candida glabrata. Mycoses 54, e39-e43.

Shuford, J. A., Rouse, M. S., Piper, K. E., Steckelberg, J. M. \& Patel, R. (2006). Evaluation of caspofungin and amphotericin B deoxycholate against Candida albicans biofilms in an experimental intravascular catheter infection model. J Infect Dis 194, 710-713.

Uppuluri, P., Dinakaran, H., Thomas, D. P., Chaturvedi, A. K. \& Lopez-Ribot, J. L. (2009). Characteristics of Candida albicans biofilms grown in a synthetic urine medium. J Clin Microbiol 47, 4078-4083.

Yeater, K. M., Chandra, J., Cheng, G., Mukherjee, P. K., Zhao, X., Rodriguez-Zas, S. L., Kwast, K. E., Ghannoum, M. A. \& Hoyer, L. L. (2007). Temporal analysis of Candida albicans gene expression during biofilm development. Microbiology 153, 2373-2385. 